\title{
The day after De-ESCALaTE and RTOG 1016 trials results
}

\author{
Ester Orlandi ${ }^{\ddagger}, 1$ \& Lisa Licitra*, $\ddagger, 2,3$ \\ ${ }^{1}$ Radiotherapy 1-2 Units, Fondazione IRCCS Istituto Nazionale dei Tumori, Milan 20133, Italy \\ ${ }^{2}$ Medical Oncology 3 Department, Fondazione IRCCS Istituto Nazionale dei Tumori, Milan 20133, Italy \\ ${ }^{3}$ University of Milan, Milan, Italy \\ *Author for correspondence: Tel.: +39 022390 3352; Fax: +39 022390 3769; lisa.licitra@istitutotumori.mi.it \\ ${ }^{\ddagger}$ Authors contributed equally
}

\begin{abstract}
"How will the results of the De-ESCALaTE and the RTOG 1016 trials influence clinical research? Given their landmark results, we feel that the deintensification concepts of the three remaining different strategies currently under evaluation, namely deintensification with induction chemotherapy, deintensification with reduced intensity-modulated radiotherapy and deintensification with mininvasive surgery, should be challenged."
\end{abstract}

First draft submitted: 26 March 2019; Accepted for publication: 2 May 2019; Published online: 26 June 2019

Keywords: cetuximab • chemotherapy • cisplatin • deintensification • oropharyngeal carcinoma • radiotherapy

Human papilloma virus (HPV)-positive oropharyngeal squamous cell carcinoma (OPSCC) is now considered a distinct disease entity from HPV-negative head and neck cancer, with a superior clinical outcome with current standard treatment approaches [1]. HPV-positive OPSCCs mostly occur in young individuals compared with their HPV-negative counterparts; thus, they can live longer with treatment-related morbidity and toxicity. In particular, full-dose cisplatinum-based chemoradiotherapy is associated with considerable toxicity [2] with potential life-threatening consequences, and increases long-term sequelae, such as xerostomia and dysphagia [3,4]. Therefore, young patients with HPV-positive OPSCC may experience life-changing side effects of the treatment affecting quality of life for several decades.

However, it is still unclear which subset of HPV-related tumors shows a better prognosis. Lower-risk subgroups within high-stage disease for which deintensified treatment may be safely considered have been clinically identified: namely, subjects with low nodal category and smoking history inferior to 10-20 pack-years.

In this scenario, de-escalation (reduction of toxicity while preserving antitumor efficacy) for favorable HPVpositive OPSCC represents a rational strategy and, indeed, it has been pursued by conducting prospective research [5].

One proposed strategy replaces cisplatin with cetuximab as the radiosensitizer. Indeed, radiotherapy can induce EGFR expression in head and neck cancers, thus contributing to acquired resistance [6]. By inhibition of EGFR, cetuximab might help overcome this resistance and induce antibody-dependent cell-mediated cytotoxicity, with a more favorable safety profile than cisplatin $[7,8]$. However, an inverse association between HPV positivity and EGFR status exists [9], and therefore EGFR inhibition by cetuximab might not be as effective as chemotherapy in HPV-positive OPC.

With these premises, two Phase III trials, the De-ESCALaTE [10] and RTOG 1016 [11], have recently investigated, for the first time, the role of a deintensification approach in HPV-positive OPC patients. In the De-ESCALaTE trial [10], with an open-label randomized controlled design, locoregionally advanced patients with low-risk HPVpositive OPC were randomly assigned to receive either intravenous cisplatin $\left(100 \mathrm{mg} / \mathrm{m}^{2}\right.$ on days 1,22 and 43 ; $\mathrm{n}=166)$ or intravenous cetuximab $\left(400 \mathrm{mg} / \mathrm{m}^{2}\right.$ loading dose followed by 7 weekly administrations of $250 \mathrm{mg} / \mathrm{m}^{2}$; $\mathrm{n}=168$ ) in addition to radiotherapy (70 Gy in 35 fractions). The primary outcome was the rate of overall (early and late) severe (grade 3-5) toxicity events at 24 months from the end of treatment: this rate did not differ significantly between treatment groups at 24 months (mean number of events per patient: 4.8, 95\% CI: 4.2-5.4 with cisplatin vs 4.8, 95\% CI: 4.2-5.4 with cetuximab; $\mathrm{p}=0.98)$; a similar result was reported for all-grade toxicity. However,

Future Medicine 
there was a significant difference between cisplatin and cetuximab in terms of 2-year overall survival (OS; 97.5 vs 89.4\%; hazard ratio [HR]: 5.0; 95\% CI: 1.7-14.7; $\mathrm{p}=0.001)$ and 2-year recurrence (6.0 vs $16.1 \%$; HR: 3.4; $95 \%$ CI: $1.6-7.2 ; \mathrm{p}=0.0007)$.

Overall, similar findings were reported in the RTOG 1016, a randomized, multicenter, noninferiority trial in advanced patients with low- or intermediate-risk (29\%) HPV-positive OPC [11]. Patients were assigned to receive either intravenous cetuximab (loading dose of $400 \mathrm{mg} / \mathrm{m}^{2} 5-7$ days before radiotherapy initiation, followed by cetuximab $250 \mathrm{mg} / \mathrm{m}^{2}$ weekly for seven doses; $\left.\mathrm{n}=399\right)$ or cisplatin $\left(100 \mathrm{mg} / \mathrm{m}^{2}\right.$ on days 1 and $\left.22 ; \mathrm{n}=406\right)$. All patients received accelerated intensity-modulated radiotherapy (70 Gy in 35 fractions over 6 weeks at six fractions per week). The primary end point was OS, with a noninferiority margin of 1.45 . At a median follow-up of 4.5 years, radiotherapy plus cetuximab did not meet the noninferiority criteria for OS (HR: 1.45; 95\% upper CI: 1.94; $\mathrm{p}=0.5056$ for noninferiority; $\mathrm{p}=00163)$. Estimated 5 -year OS was $77.9 \%(95 \%$ CI: 73.4-82.5) with cetuximab and $84.6 \%$ (95\% CI: 80.6-88.6) with cisplatin. Progression-free survival and rate of locoregional failure were poorer with cetuximab. Moreover, proportions of acute moderate-to-severe toxicity $(77.4 \%$; $95 \%$ CI: $73.0-81.5$ vs 81.7\%; 95\% CI: 77.5-85.3; $\mathrm{p}=0.1586)$ and late moderate-to-severe toxicity (16.5\%; 95\% CI: $12.9-20.7$ vs 20.4\%; 95\% CI: 16.4-24.8; $\mathrm{p}=0.1904)$ were similar between cetuximab and cisplatin.

Overall, these findings, collected in well-conducted studies, show that for patients with HPV-positive OPC cetuximab + radiotherapy is associated with shorter survival and overall comparable toxicity profile compared with cisplatin + radiotherapy. Although outcome analysis was reported with different median follow-ups, given the natural history of the disease we do not expect further events.

Therefore, cisplatin + radiotherapy should be still considered the standard of care in this setting. Moreover, the results of the De-ESCALaTE and the RTOG 1016 trials support the notion that outcomes for HPV-positive OPC patients strongly depend on the type of treatment received.

How will the results of the De-ESCALaTE and the RTOG 1016 trials influence clinical research? Given their landmark results, we feel that the deintensification concepts of the three remaining different strategies currently under evaluation, namely deintensification with induction chemotherapy, deintensification with reduced intensitymodulated radiotherapy and deintensification with mininvasive surgery, should be challenged [12].

With respect to deintensification with induction chemotherapy, in the ECOG 1308 trial [13], cetuximab was employed concomitantly to $54 \mathrm{~Gy}$ in complete responders to induction chemotherapy. Considering only low-risk patients, 2-year progression-free survival and OS were 95 and 95\%, respectively. Comparable data were observed in a second study [14], in which low-risk patients who responded to induction chemotherapy received paclitaxel with 54 Gy with locoregional failure of $2 \%$ and distant metastasis rate of $7 \%$. In a third recent trial, induction chemotherapy with three cycles of carboplatin and nab-paclitaxel was followed by response-adapted dose and volume de-escalated radiation. Low-risk OPSCC patients with response $\geq 50 \%$ received radiotherapy alone consisting of 50 Gy and those with response less than $50 \%$ but $\geq 30 \%$ received 45 Gy with concomitant chemotherapy. Similar excellent oncologic outcomes were reported [15]. Since all these studies yield comparable results in low-risk subgroups as those observed in the chemotherapy arm of the De-ESCALaTE trial [10], one may wonder whether, within a de-escalation approach, if it is worth to trade full-dose induction chemotherapy for 16-20 Gy of radiotherapy or 25 Gy with concomitant chemotherapy.

For what concerns deintensification with reduced intensity-modulated radiotherapy dose, by reducing radiotherapy total dose with or without concomitant chemotherapy as in NRG HN002 study (NCT02254278), we expect that at least locoregional controls will be affected. Last, with respect to de-intensification with mininvasive surgery, the strategy adopted in low-risk patients defined by pathological staging assumes that surgery alone is sufficient. However, in light of the data under discussion we can hardly support the idea of opting out cisplatin at least to maintain an optimal distant control.

Overall, it appears that data from RTOG 1016 and De-ESCALaTE studies may produce a domino effect on the results of ongoing deintensification trials.

One crucial issue raised by the two trials concerns the appropriate selection of low-risk patients, which cannot be excluded, based on data on Tumor, Node, Metastasis stage and smoking status.

Interestingly, recent data suggest that distinct genetic HPV-related subtypes show clinically peculiar behaviors and potentially a differentiated chemosensitivity and radiosensitivity with resulting differences in treatment response and outcome [16,17]. In particular, recently, Gleber-Netto et al. assessed data from 80 OPSCC patients in the Cancer Genome Atlas, and found a panel of $582 \mathrm{HPV}$-correlated genes, which differentiate three prognostic categories with statistically significant survival differences: OPSCC-C1 or low-risk death HPV-positive group; OPSCC-C2 
or high-risk death HPV-positive group; and OPSCC-C3 or HPV-negative group. Additional analysis discovered a panel of 38 transcripts that were able to distinguish the outcome between the two HPV-positive groups [17].

Moreover, potential targetable oncogenic alterations showed specific HPV-related complex mutational patterns that include loss of TRAF3, activating mutations of PIK3CA and amplification of E2F1 [18].

In this scenario, we would advocate that future research focuses on identifying ideal HPV-positive de-escalation candidates by exploiting tumor genetic stratification in association with clinical features [12]. We believe that by adopting a more precise approach, we may maximize the probabilities of translating research efforts into an effective de-escalation.

\section{Financial \& competing interests disclosure}

L Licitra received honoraria for public speaking in medical meetings and/or expert opinion in advisory boards from Bristol-Myers Squibb, Eisai, MSD, Merck-Serono, Boehringer Ingelheim, Novartis, AstraZeneca, Roche, Bayer, Debiopharm, Sobi, Kura Oncology, Health \& Life srl, Ipsen Innovation, Doxa Pharma srl, Amgen and Nanobiotics Sa; for clinical expertise for the approval of scientific publications from Immuno-Oncology Hub; and for public speaking and or teaching in medical meetings from AccMed and Incyte Biosciences Italy srl. She received direct research funding from AccMed, Medical Science Fundation G Lorenzini, Associazione Sinapsi, Think 2 IT, Aiom Servizi, Prime Oncology, WMA Congress (Education), Fasi, DueCi Promotion Srl, MI\&T, Net Congress \& Education and PRMA Consulting. She has received financial support from AstraZeneca S.p.A, BMS, Boehringer Ingelheim, Celgene International, Eisai, Exelixis Inc., Hoffmann-La Roche Ltd, IRX Therapeutics, Inc., Medpace Inc., Merck-Serono SpA, MSD, Novartis, Pfizer and Roche SpA. The authors have no other relevant affiliations or financial involvement with any organization or entity with a financial interest in or financial conflict with the subject matter or materials discussed in the manuscript apart from those disclosed.

Medical writing support was provided by Luca Giacomelli, and Aashni Shah, of Polistudium (Milan, Italy) and was funded by internal funds.

\section{References}

1. Castellsague X, Alemany L, Quer M. HPV involvement in head and neck cancers: comprehensive assessment of biomarkers in 3680 patients. J. Natl Cancer Inst. 108, djv403 (2016).

2. Szturz P, Wouters K, Kiyota N et al. Weekly low-dose versus three-weekly high-dose cisplatin for concurrent chemoradiation in locoregionally advanced non-nasopharyngeal head and neck cancer: a systematic review and meta-analysis of aggregate data. Oncologist 22(9), 1056-1066 (2017).

3. Frakes JM, Naghavi AO, Demetriou SK et al. Determining optimal follow-up in the management of human papillomavirus-positive oropharyngeal cancer. Cancer 122(4), 634-641 (2016).

4. Goepfert RP, Lewin JS, Barrow MP et al. Long-term, prospective performance of the MD Anderson dysphagia inventory in "low-intermediate risk" oropharyngeal carcinoma after intensity modulated radiation therapy. Int. J. Radiat. Oncol. Biol. Phys. 97(4), 700-708 (2017).

5. Mehanna H. Update on de-intensification and intensification studies in HPV. Recent Results Cancer Res. 206, 251-256 (2017)

6. Dent P, Reardon DB, Park JS. Radiation-induced release of transforming growth factor alpha activates the epidermal growth factor receptor and mitogen-activated protein kinase pathway in carcinoma cells, leading to increased proliferation and protection from radiation-induced cell death. Mol. Biol. Cell. 10, 2493-2506 (1999).

7. Bonner JA, Harari PM, Giralt J. Radiotherapy plus cetuximab for squamous-cell carcinoma of the head and neck. N. Engl. J. Med. 354, 567-578 (2006).

8. Rosenthal DI, Harari PM, Giralt J. Association of human papillomavirus and p16 status with outcomes in the IMCL-9815 Phase III registration trial for patients with locoregionally advanced oropharyngeal squamous cell carcinoma of the head and neck treated with radiotherapy with or without cetuximab. J. Clin. Oncol. 34, 1300-1308 (2016)

9. Reimers N, Kasper HU, Weissenborn SJ. Combined analysis of HPV-DNA, p16 and EGFR expression to predict prognosis in oropharyngeal cancer. Int. J. Cancer 120, 1731-1738 (2007).

10. Mehanna H, Robinson M, Hartley A et al. Radiotherapy plus cisplatin or cetuximab in low-risk human papillomavirus-positive oropharyngeal cancer (De-ESCALaTE HPV): an open-label randomised controlled Phase III trial. Lancet 393, 51-60 (2019).

11. Gillison ML, Trotti AM, Harris J et al. Radiotherapy plus cetuximab or cisplatin in human papillomavirus-positive oropharyngeal cancer (NRG Oncology RTOG 1016): a randomised, multicentre, non-inferiority trial. Lancet 393, 40-50 (2019).

12. Orlandi E, Licitra L. Personalized medicine and the contradictions and limits of first-generation deescalation trials in patients with human papillomavirus-positive oropharyngeal cancer. JAMA Otolaryngol. Head Neck Surg. 144, 99-100 (2018).

13. Marur S, Li S, Cmelak AJ et al. E1308: Phase II trial of induction chemotherapy followed by reduced-dose radiation and weekly cetuximab in patients with HPV-associated resectable squamous cell carcinoma of the oropharynx. J. Clin. Oncol. 35, 490-497 (2017). 
14. Chen AM, Felix C, Wang PC et al. Reduced-dose radiotherapy for human papillomavirus-associated squamous-cell carcinoma of the oropharynx: a single-arm, Phase 2 study. Lancet Oncol. 18, 803-811 (2017).

15. Seiwert TY, Foster CC, Blair EA et al. OPTIMA: a Phase II dose and volume de-escalation trial for human papillomavirus-positive oropharyngeal cancer. Ann. Oncol. 30(2), 297-302 (2019).

16. Wichmann G, Rosolowski M, Krohn K et al. The role of HPV RNA transcription, immune response-related gene expression and disruptive TP53 mutations in diagnostic and prognostic profiling of head and neck cancer. Int. J. Cancer 137(12), 2846-2857 (2015).

17. Gleber-Netto FO, Rao X, Guo T et al. Variations in HPV function are associated with survival in squamous cell carcinoma. JCI Insight 4(1), e124762 (2019).

18. Cancer Genome Atlas Network. Comprehensive genomic characterization of head and neck squamous cell carcinomas. Nature 517(7536), 576-582 (2015). 